\title{
On the perturbation functions and similarity orbits
}

by

\author{
HAÏKEL SKHIRI (Monastir)
}

\begin{abstract}
We show that the essential spectral radius $\varrho_{e}(T)$ of $T \in B(H)$ can be calculated by the formula $\varrho_{e}(T)=\inf \left\{\mathcal{F}_{\sharp \cdot \sharp}\left(X T X^{-1}\right): X\right.$ an invertible operator $\}$, where $\mathcal{F}_{\sharp \cdot \sharp}(T)$ is a $\Phi_{1}$-perturbation function introduced by Mbekhta [J. Operator Theory 51 (2004)]. Also, we show that if $\mathcal{G}_{\sharp \sharp \sharp}(T)$ is a $\Phi_{2}$-perturbation function [loc. cit.] and if $T$ is a Fredholm operator, then $\operatorname{dist}\left(0, \sigma_{e}(T)\right)=\sup \left\{\mathcal{G}_{\sharp} \cdot \sharp\left(X T X^{-1}\right): X\right.$ an invertible operator $\}$.
\end{abstract}

1. Terminology and introduction. Let $(H,\|\cdot\|)$ be a complex, infinite-dimensional Hilbert space and let $\mathcal{N}$ denote the set of all norms $\sharp \cdot \sharp$ on $H$ that are equivalent to $\|\cdot\|$, and derived from an inner product $\prec \cdot, \cdot \succ$ on $H$, that is, $\sharp x \sharp=\sqrt{\prec x, x \succ}$ for all $x \in H\left({ }^{1}\right)$.

Let $B(H)$ be the Banach algebra of all bounded linear operators on $H$ and let $K(H)$ be its ideal of compact operators. If $T \in B(H)$ and $\sharp \cdot \sharp \in \mathcal{N}$, we will denote by $\sharp T \sharp$ the operator-norm of $T$ relative to $\sharp \cdot \sharp$.

We denote by $N(T)$ the kernel and by $R(T)$ the range of $T \in B(H)$. The spectrum of $T$ is denoted by $\sigma(T)$, and the adjoint by $T^{*}$. An operator $T \in B(H)$ is called Fredholm (resp. semi-Fredholm) if $R(T)$ is closed and $\max \{\operatorname{dim} N(T), \operatorname{codim} R(T)\}<\infty$ (resp. $\min \{\operatorname{dim} N(T), \operatorname{codim} R(T)\}<\infty)$. We denote by $\Phi(H)$ (resp. $\Phi_{ \pm}(H)$ ) the set of all Fredholm (resp. semiFredholm) operators. Set $C(H)=B(H) / K(H)$, the Calkin algebra (see $[3,4])$; it is well known that $C(H)$ is a $C^{*}$-algebra.

The essential spectrum of $T$ is $\sigma_{e}(T)=\{\lambda \in \mathbb{C}: T-\lambda I \notin \Phi(H)\}$, and the semi-Fredholm spectrum of $T$ is $\sigma_{ \pm}(T)=\left\{\lambda \in \mathbb{C}: T-\lambda I \notin \Phi_{ \pm}(H)\right\}$. Recall that the essential spectral radius of $T$ is $\varrho_{e}(T)=\sup \left\{|\lambda|: \lambda \in \sigma_{e}(T)\right\}$.

If $T$ a semi-Fredholm operator, then the index of $T$ is defined as

$$
\operatorname{ind}(T)=\operatorname{dim} N(T)-\operatorname{codim} R(T) .
$$

2000 Mathematics Subject Classification: Primary 47A53; Secondary 47A30.

Key words and phrases: Fredholm operator, essential spectrum, essential spectral radius, Calkin algebra, similarity orbit.

$\left({ }^{1}\right)$ From the polar identity, it follows that the inner product is unique:

$$
4 \prec x, y \succ=\sharp x+y \sharp^{2}-\sharp x-y \sharp^{2}+i \sharp x+i y \sharp^{2}-i \sharp x-i y \sharp^{2} .
$$


Let $\Phi_{ \pm}^{n}$ denote the set of semi-Fredholm operators with $\operatorname{ind}(T)=n \in \mathbb{Z} \cup$ $\{+\infty,-\infty\}$. Finally, let $G(H)$ be the group of all invertible elements in $B(H)$.

The rest of this paper is organized as follows. In the next section we shall show that for a $\Phi_{1}$-perturbation function $\mathcal{F}_{\sharp \cdot \sharp}$, the infimum of $\left\{\mathcal{F}_{\sharp \cdot \sharp}\left(X T X^{-1}\right)\right.$ : $X \in G(H)\}$ is equal to $\varrho_{e}(T)$. In Section 3 we prove that if $T$ is a Fredholm operator and if $\mathcal{G}_{\sharp \sharp \sharp}(T)$ is a $\Phi_{2}$-perturbation function, then the supremum of $\left\{\mathcal{G}_{\sharp: \sharp}\left(X T X^{-1}\right): X \in G(H)\right\}$ is equal to $\operatorname{dist}\left(0, \sigma_{e}(T)\right)$.

2. Similarity orbits and $\Phi_{1}$-perturbation functions. Recently, Mbekhta [8] has introduced the following definition.

Definition 2.1 ([8, Definition 2.1]). Let $\sharp \cdot \sharp \in \mathcal{N}$. A $\Phi_{1}$-perturbation function on $B(H)$ is a function $\mathcal{F}_{\sharp \cdot \sharp}$ which associates to each $T \in B(H)$ a real number $\mathcal{F}_{\sharp \sharp \sharp}(T) \geq 0$ such that:

(a) $\mathcal{F}_{\sharp \cdot \sharp}(T+K)=\mathcal{F}_{\sharp \sharp \sharp}(T)$ for all $K \in K(H)$;

(b) $\mathcal{F}_{\sharp: \sharp}(I)=1$;

(c) $\min \left\{\mathcal{F}_{\sharp \cdot \sharp}(S T), \mathcal{F}_{\sharp \cdot \sharp}(T S)\right\} \leq \sharp S \sharp \mathcal{F}_{\sharp \cdot \sharp}(T)$ for all $T, S \in B(H)$;

(d) if $|\lambda|>\mathcal{F}_{\sharp \cdot \sharp}(T)$ then $T-\lambda I$ is Fredholm.

REMARK. The definition given by Galaz-Fontes [5] for a perturbation function is a particular case of the above definition.

From now on, we shall denote by $\mathcal{F}_{\sharp \cdot \sharp}$ a $\Phi_{1}$-perturbation function with $\sharp \cdot \sharp \in \mathcal{N}$.

In the proof of the following lemma, we use a method introduced by Mbekhta [7].

Lemma 2.2. Let $T \in B(H)$ and $\varepsilon>0$. Then there exists $W_{\varepsilon} \in B(H)$ such that

$$
\mathcal{F}_{\sharp: \sharp}\left(e^{W_{\varepsilon}} T e^{-W_{\varepsilon}}\right) \leq \varrho_{e}(T)+\varepsilon .
$$

Proof. By [10, Lemma 6], there exists a finite rank operator $K_{\varepsilon}$ such that

$$
\varrho\left(T+K_{\varepsilon}\right) \leq \varrho_{e}(T)+\varepsilon / 2 .
$$

Since $\varrho\left(\frac{T+K_{\varepsilon}}{\varrho_{e}(T)+\varepsilon}\right)<1$, it follows from the Rota theorem [12, Theorem 2] that there exists $X_{\varepsilon} \in B(H)$ invertible such that

$$
\sharp X_{\varepsilon}\left(T+K_{\varepsilon}\right) X_{\varepsilon}^{-1} \sharp \leq \varrho_{e}(T)+\varepsilon .
$$

Let $X_{\varepsilon}=U P_{\varepsilon}$ be the polar decomposition of $X_{\varepsilon}$ with $P_{\varepsilon}=\left(X_{\varepsilon}^{*} X_{\varepsilon}\right)^{1 / 2}$. Recall that $U$ is unitary, and $P_{\varepsilon}$ is positive and invertible. Since $\sigma\left(P_{\varepsilon}\right) \subseteq$ ] $0,+\infty\left[, \log\right.$ is a continuous real function on $\sigma\left(P_{\varepsilon}\right)$. It follows from the symbolic calculus that there is a self-adjoint $W_{\varepsilon} \in B(H)$ such that $P_{\varepsilon}=e^{W_{\varepsilon}}$. Thus $P_{\varepsilon}^{-1}=e^{-W_{\varepsilon}}$. Since $U$ is unitary, we see that $\sharp X_{\varepsilon}\left(T+K_{\varepsilon}\right) X_{\varepsilon}^{-1} \sharp=$ 
$\sharp e^{W_{\varepsilon}}\left(T+K_{\varepsilon}\right) e^{-W_{\varepsilon}}$. By property (a) of Definition 2.1, it follows that

$$
\mathcal{F}_{\sharp: \sharp}\left(e^{W_{\varepsilon}} T e^{-W_{\varepsilon}}\right)=\mathcal{F}_{\sharp: \sharp}\left(e^{W_{\varepsilon}}\left(T+K_{\varepsilon}\right) e^{-W_{\varepsilon}}\right) .
$$

Using properties (b) and (c) of Definition 2.1, we deduce that

$$
\begin{aligned}
\mathcal{F}_{\sharp: \sharp}\left(e^{W_{\varepsilon}}\left(T+K_{\varepsilon}\right) e^{-W_{\varepsilon}}\right) & \leq \sharp e^{W_{\varepsilon}}\left(T+K_{\varepsilon}\right) e^{-W_{\varepsilon} \sharp} \\
& \leq \sharp X_{\varepsilon}\left(T+K_{\varepsilon}\right) X_{\varepsilon}^{-1} \sharp \leq \varrho_{e}(T)+\varepsilon .
\end{aligned}
$$

Therefore, $\mathcal{F}_{\sharp: \sharp}\left(e^{W_{\varepsilon}} T e^{-W_{\varepsilon}}\right) \leq \varrho_{e}(T)+\varepsilon$.

REMARK. In the above proof, we used the notion of adjoint operator, which depends on the scalar product associated to the norm $\sharp \cdot \sharp$.

Theorem 2.3. Let $T \in B(H)$. Then

$$
\varrho_{e}(T)=\inf \left\{\mathcal{F}_{\sharp \sharp \sharp}\left(X T X^{-1}\right): X \in G(H)\right\} .
$$

Proof. First, by the property (d) of $\mathcal{F}_{\sharp \sharp \sharp}(T)$ (see Definition 2.1), for all invertible operators $X$ we have

$$
\varrho_{e}\left(X T X^{-1}\right) \leq \mathcal{F}_{\sharp \cdot \sharp}\left(X T X^{-1}\right) .
$$

Since $\varrho_{e}\left(X T X^{-1}\right)=\varrho_{e}(T)$, we obtain

$$
\varrho_{e}(T) \leq \inf \left\{\mathcal{F}_{\sharp \sharp \sharp}\left(X T X^{-1}\right): X \in G(H)\right\} .
$$

Conversely, given $\varepsilon>0$, by Lemma 2.2 there exists $W_{\varepsilon} \in B(H)$ such that

$$
\mathcal{F}_{\sharp \sharp \sharp}\left(e^{W_{\varepsilon}} T e^{-W_{\varepsilon}}\right) \leq \varrho_{e}(T)+\varepsilon .
$$

Since $e^{W_{\varepsilon}}$ is invertible, we deduce that

$$
\inf \left\{\mathcal{F}_{\sharp \cdot \sharp}\left(X T X^{-1}\right): X \in G(H)\right\} \leq \inf \left\{\varrho_{e}(T)+\varepsilon: \varepsilon>0\right\}=\varrho_{e}(T) .
$$

REMARK. If $\mathcal{F}_{\sharp \sharp \sharp}(\cdot)=\sharp \cdot \sharp_{e}$, the result we obtain is the same as in [11], when the $C^{*}$-algebra is $B(H)$ and $I=K(H)$.

From the first part of the above proof and Lemma 2.2, we obtain the following theorem.

Theorem 2.4. Let $T \in B(H)$. Then

$$
\varrho_{e}(T)=\inf \left\{\mathcal{F}_{\sharp \sharp \sharp}\left(e^{X} T e^{-X}\right): X \in B(H)\right\} .
$$

REMARK. If $\mathcal{F}_{\sharp \cdot \sharp}(\cdot)=\sharp \cdot \sharp_{e}$, we obtain the result of [9] in the particular case when the $C^{*}$-algebra is $C(H)=B(H) / K(H)$.

Theorems 2.3 and 2.4 have the following consequence.

Corollary 2.5. Let $T \in B(H)$. Then

$$
\begin{aligned}
\varrho_{e}(T) & =\inf \left\{\mathcal{F}_{\sharp: \sharp}\left(X T X^{-1}\right): X \in G(H), \sharp \cdot \sharp \in \mathcal{N}\right\} \\
& =\inf \left\{\mathcal{F}_{\sharp: \sharp}\left(e^{X} T e^{-X}\right): X \in B(H), \sharp \cdot \sharp \in \mathcal{N}\right\} .
\end{aligned}
$$


Consider the natural map $\pi: B(H) \rightarrow C(H)=B(H) / K(H)$. Let $X \in$ $\Phi(H)$. We say that $X_{\pi} \in B(H)$ is a $\pi$-inverse of $X$ if $\pi\left(X_{\pi}\right)$ is the inverse of $\pi(T)$ in $C(H)$, i.e.

$$
\pi(X) \pi\left(X_{\pi}\right)=\pi\left(X_{\pi}\right) \pi(X)=\pi(I) .
$$

From (2.1), it is easily seen that

$$
\begin{aligned}
& \sigma_{e}(T)=\sigma_{e}\left(X T X_{\pi}\right)=\sigma_{e}\left(X_{\pi} T X\right), \\
& \varrho_{e}(T)=\varrho_{e}\left(X T X_{\pi}\right)=\varrho_{e}\left(X_{\pi} T X\right) .
\end{aligned}
$$

Corollary 2.6. Let $T \in B(H)$. Then

$$
\varrho_{e}(T)=\inf \left\{\mathcal{F}_{\sharp \cdot \sharp}\left(X T X_{\pi}\right): X \in \Phi(H)\right\} .
$$

Proof. Since $G(H) \subseteq \Phi(H)$, it follows from Theorem 2.3 that

$$
\varrho_{e}(T)=\inf \left\{\mathcal{F}_{\sharp \cdot \sharp}\left(X T X^{-1}\right): X \in G(H)\right\} \geq \inf \left\{\mathcal{F}_{\sharp \cdot \sharp}\left(X T X_{\pi}\right): X \in \Phi(H)\right\} .
$$

Conversely, by the property (d) of $\mathcal{F}_{\sharp \sharp \sharp}$ (see Definition 2.1), for all $X \in \Phi(H)$ we have

$$
\varrho_{e}\left(X T X_{\pi}\right) \leq \mathcal{F}_{\sharp \cdot \sharp}\left(X T X_{\pi}\right) .
$$

The result follows from (2.4) and (2.3).

Corollary 2.7. Let $T \in B(H)$. Then

$$
\varrho_{e}(T)=\inf \left\{\mathcal{F}_{\sharp \cdot \sharp}\left(X T X_{\pi}\right): X \in \Phi(H), \sharp \cdot \sharp \in \mathcal{N}\right\} .
$$

We will show similar results for left and right invertible operators. First we need some notation. Let $G_{l}(H)$ denote the set of all left invertible operators:

$$
G_{l}(H)=\{X \in B(H): \exists L \in B(H) \text { such that } L X=I\},
$$

and $G_{r}(H)$ the set of all right invertible operators:

$$
G_{r}(H)=\{X \in B(H): \exists R \in B(H) \text { such that } X R=I\} .
$$

We shall denote by $X^{l}$ (resp. $\left.X^{r}\right)$ a left (resp. right) inverse of $X \in G_{l}(H)$ (resp. $X \in G_{r}(H)$ ).

Corollary 2.8. Let $T \in B(H)$. Then

$$
\varrho_{e}(T)=\inf \left\{\mathcal{F}_{\sharp \cdot \sharp}\left(X T X^{l}\right): X \in G_{l}(H), \operatorname{ind}(X) \in \mathbb{Z}_{-}\right\} .
$$

Proof. Since $G(H) \subseteq\left\{X \in G_{l}(H): \operatorname{ind}(X) \in \mathbb{Z}_{-}\right\} \subseteq \Phi(H)$, it follows from Theorem 2.3 and Corollary 2.6 that

$$
\begin{aligned}
\varrho_{e}(T) & =\inf \left\{\mathcal{F}_{\sharp \cdot \sharp}\left(X T X^{-1}\right): X \in G(H)\right\} \\
& \geq\left\{\mathcal{F}_{\sharp: \sharp}\left(X T X^{l}\right): X \in G_{l}(H), \operatorname{ind}(X) \in \mathbb{Z}_{-}\right\} \\
& \geq \inf \left\{\mathcal{F}_{\sharp: \sharp}\left(X T X_{\pi}\right): X \in \Phi(H)\right\}=\varrho_{e}(T) .
\end{aligned}
$$


Corollary 2.9. Let $T \in B(H)$. Then

$$
\varrho_{e}(T)=\inf \left\{\mathcal{F}_{\sharp \sharp \sharp}\left(X T X^{l}\right): X \in G_{l}(H), \operatorname{ind}(X) \in \mathbb{Z}_{-}, \sharp \cdot \sharp \in \mathcal{N}\right\} .
$$

For right invertible operators we have the following corollaries.

Corollary 2.10. Let $T \in B(H)$. Then

$$
\varrho_{e}(T)=\inf \left\{\mathcal{F}_{\sharp \cdot \sharp}\left(X T X^{r}\right): X \in G_{r}(H), \operatorname{ind}(X) \in \mathbb{N}\right\} .
$$

Corollary 2.11. Let $T \in B(H)$. Then

$$
\varrho_{e}(T)=\inf \left\{\mathcal{F}_{\sharp \sharp \sharp}\left(X T X^{r}\right): X \in G_{r}(H), \operatorname{ind}(X) \in \mathbb{N}, \sharp \cdot \sharp \in \mathcal{N}\right\} .
$$

We denote by $G_{ \pm}(H)=G_{l}(H) \cup G_{r}(H)$ the set of all semi-invertible operators. When $X \in G_{ \pm}(H)$, we simply write $X^{ \pm}$for a left inverse or a right inverse of $X$.

The proof of the following is exactly the same as the proof of Corollary 2.8 .

Corollary 2.12. Let $T \in B(H)$. Then

$$
\varrho_{e}(T)=\inf \left\{\mathcal{F}_{\sharp \sharp \sharp}\left(X T X^{ \pm}\right): X \in G_{ \pm}(H), \operatorname{ind}(X) \in \mathbb{Z}\right\} .
$$

Corollary 2.13. Let $T \in B(H)$. Then

$$
\varrho_{e}(T)=\inf \left\{\mathcal{F}_{\sharp \sharp \sharp}\left(X T X^{ \pm}\right): X \in G_{ \pm}(H), \operatorname{ind}(X) \in \mathbb{Z}, \sharp \cdot \sharp \in \mathcal{N}\right\} .
$$

3. Similarity orbits and $\Phi_{2}$-perturbation functions. We denote by $\sigma_{l}(T)=\left\{\lambda \in \mathbb{C}: T-\lambda I \notin G_{l}(H)\right\}$ the left spectrum and by $\sigma_{r}(T)=$ $\left\{\lambda \in \mathbb{C}: T-\lambda I \notin G_{r}(H)\right\}$ the right spectrum. Moreover, $\Phi_{ \pm}^{n}(T)=\{\lambda \in \mathbb{C}:$ $\left.T-\lambda I \in \Phi_{ \pm}^{n}\right\}$, with $n \in \mathbb{Z} \cup\{+\infty,-\infty\}$.

The following definition was introduced by Mbekhta [8].

Definition 3.1 ([8, Definition 3.4]). Let $\sharp \cdot \sharp \in \mathcal{N}$. A $\Phi_{2}$-perturbation function on $B(H)$ is a function $\mathcal{G}_{\sharp: \sharp}$ which associates to each $T \in B(H)$ a real number $\mathcal{G}_{\sharp: \sharp}(T) \geq 0$ such that:

(a) $\mathcal{G}_{\sharp: \sharp}(T+K)=\mathcal{G}_{\sharp \cdot \sharp}(T)$ for all $K \in K(H)$;

(b) $\mathcal{G}_{\sharp: \sharp}(I)=1$;

(c) $\min \left\{\mathcal{G}_{\sharp: \sharp}(S T), \mathcal{G}_{\sharp \cdot \sharp}(T S)\right\} \leq \sharp S \sharp \mathcal{G}_{\sharp \cdot \sharp}(T)$ for all $T, S \in B(H)$;

(d) if $T \in \Phi(H)$ and $|\lambda|<\mathcal{G}_{\sharp \sharp \sharp}(T)$, then $T-\lambda I \in \Phi(H)$.

We shall denote by $\mathcal{G}_{\sharp: \sharp}$ a $\Phi_{2}$-perturbation function with $\sharp \cdot \sharp \in \mathcal{N}$.

The following theorem is the main result of this section.

Theorem 3.2. Let $T \in \Phi(H)$. Then

$$
\operatorname{dist}\left(0, \sigma_{e}(T)\right)=\sup \left\{\mathcal{G}_{\sharp \sharp \sharp}\left(X T X^{-1}\right): X \in G(H)\right\} .
$$


For the proof we need some lemmas.

Lemma 3.3. Let $S \in B(H)$. If $\lambda_{0} \in \sigma_{e}(S)^{c} \cap \partial\left[\sigma_{l}(S)\right]$, then $\lambda_{0}$ is an isolated point of $\sigma_{l}(S)$.

Proof. The result follows from [3, Theorem 3.2.10] (see also [6, Theorem V.1.6 and Corollary V.1.7]).

Lemma 3.4. Let $T \in \Phi(H)$ and let $K$ be a compact operator such that

$$
\sigma_{e}(T)=\left[\sigma_{l}(T+K) \cap \sigma_{r}(T+K)\right] \cup \Phi_{ \pm}^{+\infty}(T) \cup \Phi_{ \pm}^{-\infty}(T) .
$$

Then $\partial\left(\sigma_{l}(T+K)\right) \cap\left[\sigma_{e}(T)\right]^{c}=\emptyset$.

Proof. Suppose there exists $\lambda_{0} \in \partial\left(\sigma_{l}(T+K)\right) \cap\left[\sigma_{e}(T)\right]^{c}$. Lemma 3.3 asserts that $\lambda_{0}$ is an isolated point of $\sigma_{l}(T+K)$. This proves that $T+K-\lambda_{0}$ is a right invertible operator, because otherwise $\lambda_{0} \in \sigma_{l}(T+K) \cap \sigma_{r}(T+K) \subseteq$ $\sigma_{e}(T)$, which is a contradiction. Now, since $T+K-\lambda_{0}$ is right invertible, we see that ind $\left(T+K-\lambda_{0} I\right) \geq 0$. But $\lambda_{0} \in \partial\left(\sigma_{l}(T+K)\right)$, which implies that $\operatorname{ind}\left(T+K-\lambda_{0} I\right)<0$, a contradiction.

Lemma 3.5. Let $T \in \Phi(H)$ and let $K$ be a compact operator as in Lemma 3.4. If $0 \notin \sigma_{l}(T+K)$, then $\operatorname{dist}\left(0, \sigma_{e}(T)\right)=\operatorname{dist}\left(0, \sigma_{l}(T+K)\right)$.

Proof. First, it is easy to see that $\partial\left[\sigma_{e}(T)\right] \subseteq \sigma_{l}(T+K) \cap \sigma_{r}(T+K)$. Therefore,

$$
\operatorname{dist}\left(0, \sigma_{e}(T)\right)=\operatorname{dist}\left(0, \sigma_{l}(T+K) \cap \sigma_{r}(T+K)\right) .
$$

We consider the case where $0 \notin \sigma_{r}(T+K)$. Since $\partial\left(\sigma_{r}(T+K)\right) \subseteq \sigma_{l}(T+K)$ and $\partial\left(\sigma_{l}(T+K)\right) \subseteq \sigma_{r}(T+K)$, we obtain

$$
\begin{aligned}
\operatorname{dist}\left(0, \sigma_{e}(T)\right) & =\operatorname{dist}\left(0, \sigma_{l}(T+K) \cap \sigma_{r}(T+K)\right) \\
& =\operatorname{dist}\left(0, \sigma_{l}(T+K)\right)=\operatorname{dist}\left(0, \sigma_{r}(T+K)\right) .
\end{aligned}
$$

On the other hand, if $0 \in \sigma_{r}(T+K)$, it was shown in Lemma 3.4 that $\partial\left(\sigma_{l}(T+K)\right) \cap \sigma_{e}(T)^{c}=\emptyset$. Thus, $\partial\left(\sigma_{l}(T+K)\right) \subseteq \sigma_{e}(T)$. Therefore,

$$
\begin{aligned}
\operatorname{dist}\left(0, \sigma_{e}(T)\right) & \leq \operatorname{dist}\left(0, \partial\left(\sigma_{l}(T+K)\right) \leq \operatorname{dist}\left(0, \sigma_{l}(T+K)\right)\right. \\
& \leq \operatorname{dist}\left(0, \sigma_{l}(T+K) \cap \sigma_{r}(T+K)\right) \leq \operatorname{dist}\left(0, \sigma_{e}(T)\right) .
\end{aligned}
$$

This proves the lemma.

Proof of Theorem 3.2. First, we show that

$$
\operatorname{dist}\left(0, \sigma_{e}(T)\right) \geq \sup \left\{\mathcal{G}_{\sharp \sharp \sharp}\left(X T X^{-1}\right): X \in G(H)\right\} \text {. }
$$

Let $X \in B(H)$ be an invertible operator, and let $\lambda \in \mathbb{C}$ be such that $|\lambda|<\mathcal{G}_{\sharp \sharp \sharp}\left(X T X^{-1}\right)$. Since $X(T-\lambda) X^{-1}=X T X^{-1}-\lambda \in \Phi(H)$, we see that $T-\lambda$ is Fredholm. Therefore,

$$
\operatorname{dist}\left(0, \sigma_{e}(T)\right) \geq \sup \left\{\mathcal{G}_{\sharp \sharp \sharp}\left(X T X^{-1}\right): X \in G(H)\right\} .
$$


Conversely, Theorem 4.5 of [1] asserts that there is $K \in K(H)$ such that $\sigma_{ \pm}(T)=\sigma_{l}(T+K) \cap \sigma_{r}(T+K)$. But

$$
\sigma_{e}(T)=\sigma_{ \pm}(T) \cup \Phi_{ \pm}^{+\infty}(T) \cup \Phi_{ \pm}^{-\infty}(T),
$$

so

$$
\sigma_{e}(T)=\left[\sigma_{l}(T+K) \cap \sigma_{r}(T+K)\right] \cup \Phi_{ \pm}^{+\infty}(T) \cup \Phi_{ \pm}^{-\infty}(T) .
$$

Since $0 \notin \sigma_{e}(T)$, we obtain $0 \notin \sigma_{l}(T+K)$ or $0 \notin \sigma_{r}(T+K)$. We will suppose that $0 \notin \sigma_{l}(T+K)$; the other case is similar. It was shown in Lemma 3.5 that $\operatorname{dist}\left(0, \sigma_{e}(T)\right)=\operatorname{dist}\left(0, \sigma_{l}(T+K)\right)$. Corollary 2.6 of [2] implies that

$(*) \quad \operatorname{dist}\left(0, \sigma_{e}(T)\right)=\operatorname{dist}\left(0, \sigma_{l}(T+K)\right)=\sup \{1 / \varrho(S): S(T+K)=I\}$.

On the other hand, let $S \in B(H)$ be a left inverse of $T+K$ and let $\varepsilon>0$. Since $\varrho\left(\frac{S}{\varrho(S)+\varepsilon}\right)<1$, it follows from the Rota theorem [12, Theorem 2] that there exists an invertible operator $Z_{\varepsilon}$ such that

$$
\sharp Z_{\varepsilon} S Z_{\varepsilon}{ }^{-1} \sharp \leq \varrho(S)+\varepsilon .
$$

Consider the polar decomposition $Z_{\varepsilon}=U P_{\varepsilon}$, where $P_{\varepsilon}=\left(Z_{\varepsilon}^{*} Z_{\varepsilon}\right)^{1 / 2}$ and $U$ is the partial isometry with $N(U)=N\left(Z_{\varepsilon}\right)$ and $R(U)=R\left(Z_{\varepsilon}\right)$. This implies that $U$ is unitary. Recall that $P_{\varepsilon}$ is positive and invertible. Since $\left.\sigma\left(P_{\varepsilon}\right) \subseteq\right] 0,+\infty\left[, \log\right.$ is a continuous real function on $\sigma\left(P_{\varepsilon}\right)$. It follows from the symbolic calculus that there is a self-adjoint $W_{\varepsilon} \in B(H)$ such that $P_{\varepsilon}=e^{W_{\varepsilon}}$. Thus $P_{\varepsilon}^{-1}=e^{-W_{\varepsilon}}$. It is obvious that

$$
\left[e^{W_{\varepsilon}}(T+K) e^{-W_{\varepsilon}}\right]\left[e^{W_{\varepsilon}} S e^{-W_{\varepsilon}}\right]\left[e^{W_{\varepsilon}}(T+K) e^{-W_{\varepsilon}}\right]=e^{W_{\varepsilon}}(T+K) e^{-W_{\varepsilon}} .
$$

It follows from [8, Lemme 3.18] that

$$
\mathcal{G}_{\sharp \sharp \sharp}\left(e^{W_{\varepsilon}} T e^{-W_{\varepsilon}}\right)=\mathcal{G}_{\sharp \sharp \sharp}\left(e^{W_{\varepsilon}}(T+K) e^{-W_{\varepsilon}}\right) \geq \frac{1}{\sharp e^{W_{\varepsilon}} S e^{-W_{\varepsilon} \sharp e}} .
$$

But $\sharp e^{W_{\varepsilon}} S e^{-W_{\varepsilon} \sharp} \geq \sharp e^{W_{\varepsilon}} S e^{-W_{\varepsilon}} \sharp e$, so

$$
\mathcal{G}_{\sharp \sharp \sharp}\left(e^{W_{\varepsilon}} T e^{-W_{\varepsilon}}\right) \geq \frac{1}{\sharp e^{W_{\varepsilon}} S e^{-W_{\varepsilon} \sharp}} .
$$

Since $Z_{\varepsilon}=U P_{\varepsilon}=U e^{W_{\varepsilon}}$ and $U$ is a unitary operator, we deduce that

$$
\mathcal{G}_{\sharp \sharp \sharp}\left(e^{W_{\varepsilon}} T e^{-W_{\varepsilon}}\right) \geq \frac{1}{\sharp Z_{\varepsilon} S Z_{\varepsilon}^{-1_{\sharp}}} .
$$

It follows from $(* *)$ that

$$
\sup _{\varepsilon>0}\left\{\mathcal{G}_{\sharp \sharp \sharp}\left(e^{W_{\varepsilon}} T e^{-W_{\varepsilon}}\right)\right\} \geq \sup _{\varepsilon>0}\left\{\frac{1}{\sharp Z_{\varepsilon} S Z_{\varepsilon}^{-1} \sharp}\right\} \geq \frac{1}{\varrho(S)} .
$$

But

$$
\sup \left\{\mathcal{G}_{\sharp: \sharp}\left(X T X^{-1}\right): X \in G(H)\right\} \geq \sup _{\varepsilon>0}\left\{\mathcal{G}_{\sharp: \sharp}\left(e^{W_{\varepsilon}} T e^{-W_{\varepsilon}}\right)\right\} .
$$


We deduce that

$$
\sup \left\{\mathcal{G}_{\sharp \sharp \sharp}\left(X T X^{-1}\right): X \in G(H)\right\} \geq 1 / \varrho(S) .
$$

Since $(* * *)$ holds for all left inverses of $T+K$, we obtain

$$
\sup \left\{\mathcal{G}_{\sharp \cdot \sharp}\left(X T X^{-1}\right): X \in G(H)\right\} \geq \sup \{1 / \varrho(S): S(T+K)=I\} .
$$

It follows from $(*)$ that

$$
\sup \left\{\mathcal{G}_{\sharp \cdot \sharp}\left(X T X^{-1}\right): X \in G(H)\right\} \geq \operatorname{dist}\left(0, \sigma_{e}(T)\right) .
$$

It is easy to see that the above proof yields the following result.

Theorem 3.6. Let $T \in \Phi(H)$. Then

$$
\operatorname{dist}\left(0, \sigma_{e}(T)\right)=\sup \left\{\mathcal{G}_{\sharp: \sharp}\left(e^{X} T e^{-X}\right): X \in B(H)\right\} .
$$

Corollary 3.7. Let $T \in \Phi(H)$. Then

$$
\begin{aligned}
\operatorname{dist}\left(0, \sigma_{e}(T)\right) & =\sup \left\{\mathcal{G}_{\sharp \cdot \sharp}\left(X T X^{-1}\right): X \in G(H), \sharp \cdot \sharp \in \mathcal{N}\right\} \\
& =\sup \left\{\mathcal{G}_{\sharp \cdot \sharp}\left(e^{X} T e^{-X}\right): X \in B(H), \sharp \cdot \sharp \in \mathcal{N}\right\} .
\end{aligned}
$$

Corollary 3.8. Let $T \in \Phi(H)$. Then

$$
\operatorname{dist}\left(0, \sigma_{e}(T)\right)=\sup \left\{\mathcal{G}_{\sharp: \sharp}\left(X T X_{\pi}\right): X \in \Phi(H)\right\} .
$$

Proof. Let $X \in \Phi(H)$ and let $\lambda \in \mathbb{C}$ be such that

$$
|\lambda|<\mathcal{G}_{\sharp \cdot \sharp}\left(X T X_{\pi}\right) \text {. }
$$

It follows from the fact that $X(T-\lambda) X_{\pi}=X T X_{\pi}-\lambda X X_{\pi} \in \Phi(H)$ and the relation (2.2) that $T-\lambda \in \Phi(H)$. Then by Theorem 3.2,

$$
\begin{aligned}
\operatorname{dist}\left(0, \sigma_{e}(T)\right) & \geq \sup \left\{\mathcal{G}_{\sharp: \sharp}\left(X T X_{\pi}\right): X \in \Phi(H)\right\} \\
& \geq \sup \left\{\mathcal{G}_{\sharp: \sharp}\left(X T X^{-1}\right): X \in G(H)\right\} \geq \operatorname{dist}\left(0, \sigma_{e}(T)\right) .
\end{aligned}
$$

Corollary 3.9. Let $T \in \Phi(H)$. Then

$$
\operatorname{dist}\left(0, \sigma_{e}(T)\right)=\sup \left\{\mathcal{G}_{\sharp \sharp \sharp}\left(X T X_{\pi}\right): X \in \Phi(H), \sharp \cdot \sharp \in \mathcal{N}\right\} .
$$

Corollary 3.10. Let $T \in \Phi(H)$. Then

$$
\operatorname{dist}\left(0, \sigma_{e}(T)\right)=\sup \left\{\mathcal{G}_{\sharp: \sharp}\left(X T X^{l}\right): X \in G_{l}(H), \operatorname{ind}(X) \in \mathbb{Z}_{-}\right\} .
$$

Proof. We deduce from Corollary 3.8 that

$$
\begin{aligned}
\operatorname{dist}\left(0, \sigma_{e}(T)\right) & =\sup \left\{\mathcal{G}_{\sharp \cdot \sharp}\left(X T X_{\pi}\right): X \in \Phi(H)\right\} \\
& \geq \sup \left\{\mathcal{G}_{\sharp \cdot \sharp}\left(X T X^{l}\right): X \in G_{l}(H), \operatorname{ind}(X) \in \mathbb{Z}_{-}\right\} .
\end{aligned}
$$

By Theorem 3.2, we conclude that

$$
\begin{aligned}
\operatorname{dist}\left(0, \sigma_{e}(T)\right) & =\sup \left\{\mathcal{G}_{\sharp \sharp \sharp}\left(X T X^{-1}\right): X \in G(H)\right\} \\
& \leq \sup \left\{\mathcal{G}_{\sharp: \sharp}\left(X T X^{l}\right): X \in G_{l}(H), \operatorname{ind}(X) \in \mathbb{Z}_{-}\right\} .
\end{aligned}
$$


We also have the following corollary.

Corollary 3.11. Let $T \in \Phi(H)$. Then

$$
\operatorname{dist}\left(0, \sigma_{e}(T)\right)=\sup \left\{\mathcal{G}_{\sharp \sharp \sharp}\left(X T X^{l}\right): X \in G_{l}(H), \operatorname{ind}(X) \in \mathbb{Z}_{-}, \sharp \cdot \sharp \in \mathcal{N}\right\} \text {. }
$$

For right invertible operators we have the following corollaries.

Corollary 3.12. Let $T \in \Phi(H)$. Then

$$
\operatorname{dist}\left(0, \sigma_{e}(T)\right)=\sup \left\{\mathcal{G}_{\sharp \cdot \sharp}\left(X T X^{r}\right): X \in G_{r}(H), \operatorname{ind}(X) \in \mathbb{N}\right\} .
$$

Corollary 3.13. Let $T \in \Phi(H)$. Then

$$
\operatorname{dist}\left(0, \sigma_{e}(T)\right)=\sup \left\{\mathcal{G}_{\sharp: \sharp}\left(X T X^{r}\right): X \in G_{r}(H), \operatorname{ind}(X) \in \mathbb{N}, \sharp \cdot \sharp \in \mathcal{N}\right\} .
$$

The proof of the following corollary is exactly the same as the proof of Corollary 3.10.

Corollary 3.14. Let $T \in \Phi(H)$. Then

$$
\operatorname{dist}\left(0, \sigma_{e}(T)\right)=\sup \left\{\mathcal{G}_{\sharp: \sharp}\left(X T X^{ \pm}\right): X \in G_{ \pm}(H), \operatorname{ind}(X) \in \mathbb{Z}\right\} .
$$

We easily obtain the following.

Corollary 3.15. Let $T \in \Phi(H)$. Then

$$
\operatorname{dist}\left(0, \sigma_{e}(T)\right)=\sup \left\{\mathcal{G}_{\sharp \sharp \sharp}\left(X T X^{ \pm}\right): X \in G_{ \pm}(H), \operatorname{ind}(X) \in \mathbb{Z}, \sharp \cdot \sharp \in \mathcal{N}\right\} \text {. }
$$

Acknowledgements. I am grateful to Professor M. Mbekhta for helpful conversations and I thank the referee for valuable remarks which improved the first draft of this paper.

\section{References}

[1] C. Apostol, The correction by compact perturbation of the singular behavior of operators, Rev. Roumaine Math. Pures Appl. 21 (1976), 155-175.

[2] C. Badea and M. Mbekhta, Compressions of resolvents and maximal radius of regularity, Trans. Amer. Math. Soc. 351 (1999), 2949-2960.

[3] S. R. Caradus, W. E. Pfaffenberger and B. Yood, Calkin Algebras and Algebras of Operators on Banach Spaces, Dekker, New York, 1974.

[4] R. G. Douglas, Banach Algebra Technique in Operator Theory, Academic Press, New York, 1972.

[5] F. Galaz-Fontes, Measures of noncompactness and upper semi-Fredholm perturbation theorems, Proc. Amer. Math. Soc. 118 (1993), 891-897.

[6] S. Goldberg, Unbounded Linear Operators, McGraw-Hill, New York, 1966.

[7] M. Mbekhta, Formules de distance au spectre généralisé et au spectre semi-Fredholm, J. Funct. Anal. 194 (2002), 231-247.

[8] - Fonctions perturbation et formules du rayon spectral essentiel et de distance au spectre essentiel, J. Operator Theory 51 (2004), 3-18.

[9] G. J. Murphy and T. T. West, Spectral radius formulae, Proc. Edinburgh Math. Soc. (2) 22 (1979), 271-275.

[10] R. D. Nussbaum, The radius of the essential spectrum, Duke Math. J. 37 (1970), 473-478. 
[11] V. Rakočević, Spectral radius formulae in quotient $C^{*}$-algebras, Proc. Amer. Math. Soc. 113 (1991), 1039-1040.

[12] G. C. Rota, On models for linear operators, Comm. Pure Appl. Math. 13 (1960), 469-472.

Département de Mathématiques

Faculté des Sciences de Monastir

Avenue de l'environnement

5019 Monastir, Tunisie

E-mail: haikel.skhiri@gmail.com, haikel.skhiri@fsm.rnu.tn

Received May 6, 2007

Revised version April 15, 2008 